\title{
ANEURISMA DE HIPERFLUXO PRÓPRIO DA ARTÉRIA COMUNICANTE POSTERIOR DECORRENTE DE EFEITO HEMODINÂMICO EM PACIENTE COM OCLUSÃO DA ARTÉRIA SUBCLÁVIA TRATADO POR ANGIOPLASTIA
}

\author{
José Maria Modenesi Freitas' ${ }^{1}$, Marcos Antônio Pieruccetti', \\ Guilherme Cabral de Andrade², Ségio Listik², Ricardo J. Costa², \\ José Carlos Rodrigues Jr3, Luis F. Haikel Jr3, \\ Marcos Rogério Gregorini3 ${ }^{3}$ Clemente Augusto B. Pereira ${ }^{4}$
}

\begin{abstract}
RESUMO - Aneurismas próprios da artéria comunicante posterior são extremamente raros possuindo uma incidência que varia de 0,1 a $2,8 \%$ de todos os aneurismas. $O$ surgimento de aneurisma intracraniano em virtude de alterações de fluxo por oclusão arterial é descrito na literatura. Apresentamos o caso de homem de 69 anos, vítima de hemorragia subaracnóidea, com diagnóstico de aneurisma próprio da artéria comunicante posterior direita. Havia também, oclusão da artéria subclávia esquerda com roubo de fluxo da artéria vertebral direita pela vertebral esquerda. Realizou-se tratamento endovascular com angioplastia e colocação de stent na artéria subclávia esquerda com conseqüente oclusão do aneurisma. Discutimos o restabelecimento do fluxo arterial intracraniano como forma de tratamento deste aneurisma.
\end{abstract}

PALAVRAS-CHAVE: aneurisma próprio, artéria comunicante posterior, oclusão arterial.

\begin{abstract}
High flow"true" posterior communicating artery aneurysm due to hemodynamic effect in a patient with subclavian artery occlusion treated with angioplasty

ABSTRACT - True posterior communicating art e ry aneurysms are extremely rare with incidence ranges from $0.1 \%$ to $2.8 \%$ of all aneurysms. Cerebral aneurysm formation has been reported as a complication of artery occlusion by flow alteration. We present a 69 years old male patient presenting with subarachnoid hemomage with diagnosis of true right posterior communicating artery aneurysm. He had a left subclavian arte ry occlusion with flow theft from the right vertebral art e ry to the left vertebral art ery. The patient underwent endovascular treatment with angioplasty and stent placement on the left subclavian artery and aneurysm occlusion result. We discuss the restablishment of cerebral blood flow as a treatment for this aneurysm.
\end{abstract}

KEY WORDS: true aneurysm, posterior communicating artery, artery occlusion.

Aneurismas da artéria carótida interna no segmento próximo a artéria comunicante posterior, assim como da junção da artéria carótida com a comunicante posterior, são tradicionalmente nomeados como aneurismas da região da comunicante posterior ${ }^{1}$. Os aneurismas próprios da artéria comunicante posterior são de ocorrência rara, variando de 0,1 a 2,8\% de todos os aneurismas intracranianos ${ }^{2,3}$. Após observação da anatomia microcirurgica, Krayenbühl e col. ${ }^{4}$, em 1972, desc reveram 4 tipos de aneurismas da artéria comunicante posterior: a) aneurisma da junção da art éria carótida interna e artéria comunicante poste- rior; b) aneurisma fusiforme da artéria comunicante posterior; c) aneurisma sacular da artéria comunicante posterior; e d) aneurisma da artéria carótida interna. O termo aneurisma próprio da artéria comunicante posterior foi usado inicialmente em 1979 por Yoschida e col. ${ }^{5}$, para determinar os aneurismas que possuem origem direta da artéria comunicante posterior, aproximadamente 2 a $3 \mathrm{~mm}$ distal à junção com a artéria carótida interna. A anatomia vascular regional da artéria comunicante posterior é importante pela presença de ramos perfurantes que darão suprimento sanguíneo ao quiasma óptico, nervo oculomotor, corpo mamilar,

Serviço de Neurocinurgia e Neurorradiologia Intervencionista do Hospital Heliópolis São Paulo SP, Brasil: ${ }^{1}$ Neuroradiologista Intervencionista; ${ }^{2}$ Neurocirurgião; ${ }^{3}$ Residente em Neurocirurgia; ${ }^{4}$ Chefe do Serviço de Neurocirurgia.

Recebido 16 Dezembro 2004, recebido na forma final 3 Março 2005. Aceito 18 Abril 2005.

Dr. Guilherme Cabral de Andrade - Rua Monte Alegre 58 / 134 - 05014-000 São Paulo SP - Brasil. E-mail: cabralnec@uol.com.br 
tuber cinéreo, crura cerebral, tálamo anterior e porção rostral do núcleo caudado ${ }^{5,6}$.

O surgimento de aneurisma cerebral por alterações hemodinâmicas, tem sido descrito em modelos experimentais 7,8 , assim como após oclusão da artéria carótida interna ${ }^{9,10}$.

\section{CASO}

Homem de 69 anos apresentou quadro clínico de cefaléia súbita seguida de perda momentânea da consciência em 20/03/2004. Após 18 dias do evento foi encaminhado ao nosso serviço. À admissão queixava-se de cefaléia; ao exame físico geral apresentava diferença na medida da pressão arterial (PA) dos membros superiores com PA de $140 \times 110 \mathrm{mmHg}$ em MSE e $170 \times 100$ $\mathrm{mmHg}$ em MSD. Ao exame neurológico apresentava-se consciente e orientado e com paresia dos nervos cranianos III (oculumotor) e IV (troclear) à direita. Realizou exame de tomografia computadorizada de crânio, que evidenciava hemorragia sub-aracnóide Fisher III (Fig 1A). À angiografia digital realizada em 28/05/2004, no arco aórtico e nas artérias carótidas e vertebrais, foi diagnosticado aneurisma sacular próprio da artéria comunicante posterior direita (Fig 1B e 1C), oclusão da artéria subclávia esquerda (Fig $2 \mathrm{~A}$ ) e roubo de fluxo da artéria ver- tebral direita pela artéria vertebral esquerda (FIG 2B), além de estenose maior que $80 \%$ na emergência da a rtéria carótida interna direita. A discussão terapêutica foi baseada no fato de que o roubo da artéria subclávia era de tamanha importância que não havia contrastação do segmento superior da artéria basilar e dos seus ramos, e que este segmento seria suprido pela artéria comunicante posterior direita. Logo a possibilidade do aneurisma ter surgido em decorrência do alto fluxo por esta artéria, mesmo com a evidência de estenose importante na carótida interna direita, foi postulada. No entanto a hipótese de que a restauração do fluxo normal, com correção do roubo da artéria subclávia pela desobstrução do segmento ocluído da artéria subclávia esquerda, eliminaria o fluxo pelas artérias comunicante posteriores, havendo a possibilidade de exclusão do aneurisma da circulação por diminuição do fluxo. Em 08/06/2004 foi realizado recanalização por ATP (artériotromboplastia) e colocação de stent na artéria subclávia esquerda (Fig $3 \mathrm{~A}$ ), restaurando o fluxo sanguíneo pela a rtéria vertebral esquerda (Fig $3 A$ ), e enchimento de toda a árv o rearterial vért e b ro-basilar e das artérias cerebrais posteriores (Fig 3B) e diminuição do fluxo pela artéria comunicante posterior com estagnação de contraste no interior do aneurisma (Fig 3C).

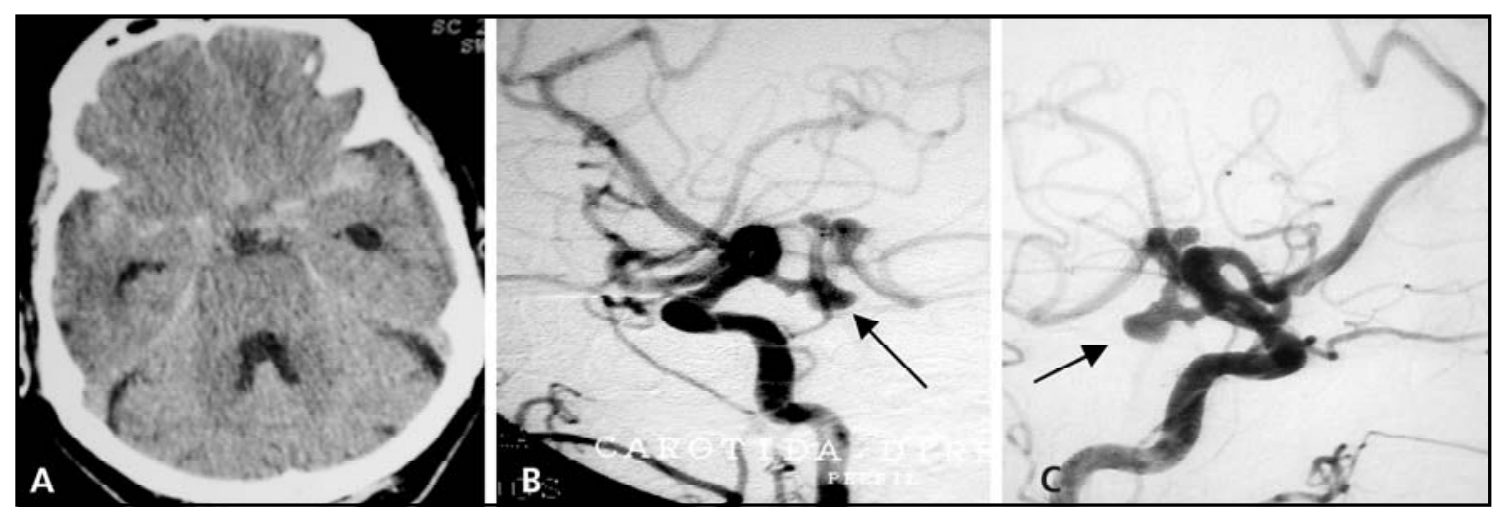

Fig 1. A) Corte axial de tomografia computadorizada evidenciando hemorragia subaracnóidea, Fisher III. B) e C) Angiografia carotídea direita mostrando aneurisma sacular próprio da artéria comunicante posterior(setaspretas).

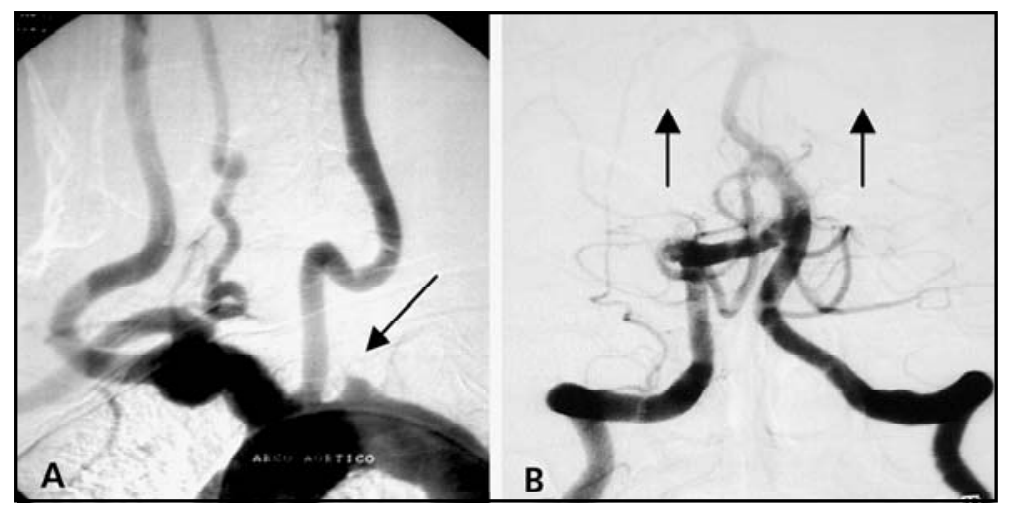

Fig 2. A) Angiografia do arco aórtico mostrando oclusão da artéria subclávia esquerda (seta preta). B) Angiografia da artéria vertebral direita mostran do roubo de fluxo da artéria vertebral direita pela esquerda e hipofluxo da artéria basilar e artérias cerebrais posteriores (setas pretas). 


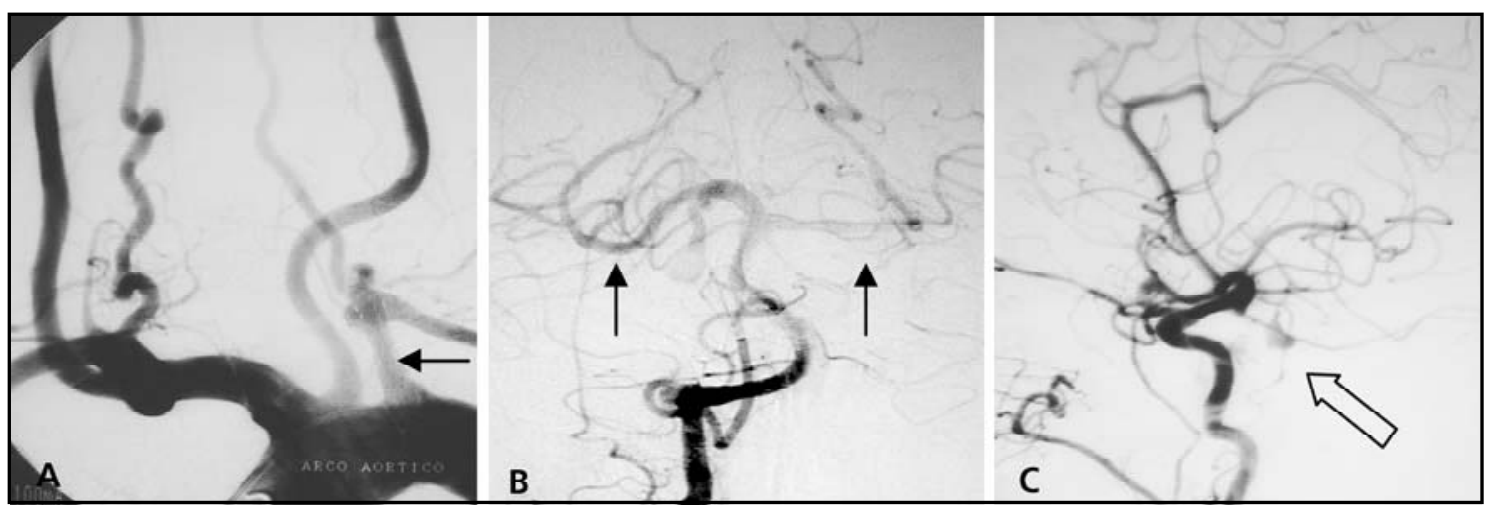

Fig 3. A) Angiografia do arco aórtico com procedimento de ATP e colocação de stent da artéria subclávia esquerda(seta p reta). B) Angiografia da artéria vertebral logo após a ATP com enchimento do sistema vérte brobasilar e das artérias ce rebrais posteriores. C) Angiografia da artéria carótida direita logo após a ATP com diminuição de fluxo pela artéria comunicante posterior e estagnação de contraste no interior do aneurisma(seta preta vazada).

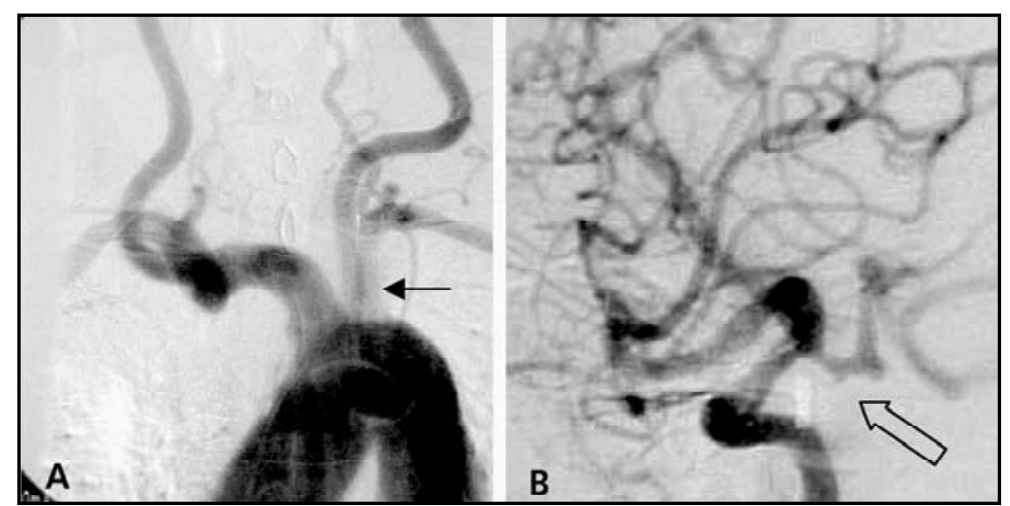

Fig 4. A) Angiografia de controle tardio, do arco a órtico e carótida direita, evidenciando permeabili dade da angioplastia da artéria subclávia esquerda (seta preta). B) Exclusão do saco aneurismático (seta preta vazada).
Em 01/09/04 o paciente apresentava-se em bom estado clínico e neurológico, com PA de $150 \times 100 \mathrm{mmHg}$ e pulsos simétricos em ambos os membros superiores. O estudo angiográfico de controle mostrou a patência da angioplastia da artéria subclávia esquerda com fluxo anterógrado na artéria vertebral esquerda (Fig 4A) e a angiografia carotídea mostrou a artéria comunicante posterior direita permeável, com exclusão do aneurisma (Fig 4B).

\section{DISCUSSÃO}

Aneurisma próprio da artéria comunicante posterior é um evento raro variando de 0,1 a 2,8\% ${ }^{2,5}$, sendo alguns considerados como aneurismas de hiperfluxo ${ }^{11,12}$. O aneurisma próprio da artéria comunicante posterior possui considerações cirúrgicas especificas em virtude da presença de vários ramos p e rfurantes para estruturas vitais ${ }^{5,6}$, assim como a presenação do nervo oculomotor. Akimura ${ }^{13} \mathrm{p}$ re coniza a realização de trapping, com o intuito de preservar principalmente os ramos tálamo estriados. O estudo hemodinâmico do arco aórtico e de todo o polígono de Willis deve ser realizado, pela possibilidade do aneurisma ser formado por alteração de fluxo. Kaspera ${ }^{12}$ demonstrou, através de Doppler transcraniano, o aumento da velocidade e turbulência do fluxo sanguíneo na artéria comunicante posterior, em paciente com oclusão de a rtéria carótida interna que desenvolveu aneurisma próprio da artéria comunicante posterior. A alteração de fluxo ocorreu neste caso, em virtude da oclusão da artéria subclávia esquerda, com roubo de fluxo da artéria vertebral direita pela vertebral esquerda e conseqüente aumento de fluxo da artéria comunicante posterior direita, causando a formação de aneurisma sacular. Para o tratamento deste aneurisma optou-se pela correção do fluxo arterial da artéria subclávia ocluída, através de ATP e colocação de stent. Houve restauração do padrão circulatório normal e diminuição do fluxo na artéria comunicante posterior, com estagnação do contraste no interior do saco aneurismático observado em angiografia de controle imediatamente após o procedimento, levando a trombose espontânea do mesmo e sem que houvesse risco de recanali- 
zação, já que entendemos que a sua fisiopatologia por alteração de fluxo difere da dos demais aneurismas, havendo fragilidade da camada elástica interna e não sua descontinuidade. O controle angiográfico tardio, comprovou a exclusão do aneurisma.

Este caso confirma que alterações da hemodinâmica do polígono de Willis podem estar relacionadas à formação de aneurismas intracranianos. A análise hemodinâmica cerebral é fundamental para a realização de procedimentos eficazes com baixa morbidade. O tratamento do aneurisma próprio da artéria comunicante posterior foi obtido. A angioplastia e colocação de stent na artéria subclávia esquerda proporcionaram a recanalização arterial, eliminando o roubo de fluxo da artéria subclávia esquerda. A alteração da hemodinâmica do polígono de Willis permitiu a exclusão espontânea do aneurisma.

\section{REFERÊNCIAS}

1. Peerless SJ.The surgical approach to middle cerebral and posterior communicating aneurysms.Clin Neurosurg 1974;21:151-164.
2. Kudo T. An operative complication in a patient with a true posterior communicating artery aneurysm: case report and review of the literature. Neurosurgery 1990;27:650-653.

3. Muneda K, Yoshizu H, Terada H.True posterior communicating artery aneurysm.No Shinkei Geka 2001;29:163-168.

4. Krayenbühl HÁ, Yasargil G,Flamm ES, Tew JM.Microsurgical treatment of intracranial saccular aneurysms.J Neurosurg 1972;37:678-686.

5. Yoschida M, Watanabe M, Kuramoto S. "True” posterior communicating artery aneurysm. Surg Neurol 1979;11:379-381.

6. Gibo H, Lenkey C, RhotonAL.Microsu rical anatomy of the supraclinoid portion of the internal carotid artery. J Neuro s u rg 1981;55: 560-574.

7. Hashimoto N, Handa H, Hazama F. Experimentally induced cerebral aneurysms in rats.Surg Neurol 1978;10:3-8.

8. German WJ, Black SPW. Experimental production of carotid aneurysms. N Engl J Med 1954;250:104-106.

9. Timperman PE, Tomsick TA, Tew JM Jr, van Loveren RH. Aneurysm formation after carotid occlusion. AJNR 1995;16:329-331.

10. Dyste GW, Beck DW.De novo aneurysm formation following carotid ligation: case report and review of the literature.Neurosurg 1989;24: 88-92.

11. Ogasawara K, Numagami $Y$, Kitahara M.A case of ruptured true posterior communicating artery aneurysm thirteen years after surgical occlusion of the ipsilateral cervical internal carotid artery. N Shinkei Geka 1995;23:359-363.

12. Kaspera W, Majchrzak H, Kopera M, Ladzinski P. "True" aneurysm of the posterior communicanting artery as a possible effect of collateral ciralation in a patient with occlusion of the internal carotid artery: a case and literature review.Minim Invasive Neuro su rg2002;45:240-244.

13. Akimura $\mathrm{T}, \mathrm{Abiko} \mathrm{S}$, Ito H.True posterior communicating artery aneurysm. Acta Neurol Scand 1991;84:207-209. 